Review Article

\title{
A Meta-Analysis of Efficacy and Safety of Infliximab for Prevention of Postoperative Recurrence in Patients with Crohn's Disease
}

\author{
He Huang, ${ }^{1}$ Su Xu, ${ }^{2}$ Fubin Huang, ${ }^{1}$ Xia Wang, ${ }^{1}$ Yong Chen, ${ }^{1}$ and Zhaoshan $X u\left(\mathbb{D}{ }^{1}\right.$ \\ ${ }^{1}$ Department of Gastroenterology, Yancheng Hospital of Traditional Chinese Medicine affiliated to Nanjing University of \\ Chinese Medicine, Yancheng 224001, China \\ ${ }^{2}$ Department of Anorectal Surgery, Yancheng Hospital of Traditional Chinese Medicine affiliated to Nanjing University of \\ Chinese Medicine, Yancheng 224001, China \\ Correspondence should be addressed to Zhaoshan Xu; xzs189@163.com
}

Received 21 May 2018; Accepted 13 August 2018; Published 13 December 2018

Academic Editor: Hiroshi Tanaka

Copyright (C) 2018 He Huang et al. This is an open access article distributed under the Creative Commons Attribution License, which permits unrestricted use, distribution, and reproduction in any medium, provided the original work is properly cited.

\begin{abstract}
Objective. We sought to investigate the efficacy and safety of Infliximab for prevention of postoperative recurrence in patients with Crohn's disease (CD), in a meta-analysis of clinical trial results. Methods. The Medline, Embase, PubMed, and Web of Science databases were systematically searched for suitable studies. A meta-analysis of enrolled studies was performed to analyze the efficacy of Infliximab on outcomes regarding the prevention of postoperative recurrence of CD. A Galbraith radial plot was used to quantify the heterogeneity. Funnel plot and Egger test were performed to describe the bias of publication. A Forest plot was prepared to indicate the efficacy outcomes. Results. A total of 7 prospective trials were included in our meta-analysis $(\mathrm{N}=455)$. The Funnel plot and Egger test showed there was no significant bias in the included publications. The Cochrane collaboration tool indicated that all 7 prospective trials were of high quality. The results of Galbraith radial plot showed that no study was the source of heterogeneity. Compared with the placebo group, Infliximab decreased the rates of endoscopic recurrence $(\mathrm{RR}=0.421 ; 95 \% \mathrm{CI} 0.328$ to $0.539 ; p<0.001)$, and there was a significant reduction in rates of clinical recurrence in the Infliximab-treated group $(\mathrm{RR}=0.519$; 95\% CI 0.349 to $0.774 ; p=0.001$ ). Furthermore, Infliximab treatment did not show adverse effects as other systematic therapeutic drugs, indicating that Infliximab treatment is effective and well tolerated. Conclusion. Compared with the controls, Infliximab is a promising therapeutic agent for the management of CD patients.
\end{abstract}

\section{Introduction}

Crohn's disease (CD), a chronic inflammatory disorder occurred in gastrointestinal tract that may cause serious tissue damage, is one of inflammatory bowel diseases [1]. $\mathrm{CD}$ usually significantly affects the patients' quality of life. In some severe cases, $\mathrm{CD}$ poses challenges for the entire family and may lead to psychological problems, even a substantial economic burden, as it frequently occurs in young patients [2]. At present, over $75 \%$ of $\mathrm{CD}$ patients need surgical resection for penetrating and structuring complications, and ileocolonic resection is the most common therapeutic strategy for such condition [3].
However, surgery is not once for all and CD patients often have clinical or endoscopic recurrence within 2-6 years of resection in up to $41 \%$ and $89 \%$, respectively [4]. Some studies have proved the efficacy of traditional drugs in the prevention of postoperative recurrence of $\mathrm{CD}$, with different results [58].

Owing to the efficacy of biological treatment in the induction and maintenance of $\mathrm{CD}$, multiple studies have been conducted to find out the efficacy of antitumor necrosis factor (anti-TNF) agents in the prevention of postoperative recurrence in CD [9-13]. The anti-TNF agents were in fact shown to be more effective than conventional treatment in the prevention of postoperative clinical and endoscopic 
recurrence [14-18]. Furthermore, three recent meta-analyses have put forward the conclusion above [19-21]. However, there are few studies researching the efficacy of Infliximab, one of the anti-TNF agents, for the prevention of postoperative recurrence in $\mathrm{CD}$, and there is currently no conclusive evidence of the superiority of Infliximab therapy in this setting. Hence, a meta-analysis of prospective trials was performed to compare the efficacy and safety of Infliximab in the prevention of postoperative recurrence of $\mathrm{CD}$ after ileocolonic resection.

In the present study, we sought to analyze the efficacy and safety of Infliximab for prevention of recurrence in patients with CD in a meta-analysis of clinical trial results. Suitable trials were selected by searching the PubMed, Medline, Embase, and Web of Science databases; only prospective trials were included. The results revealed that Infliximab treatment yielded a significant improvement in the rates of $\mathrm{CD}$ recurrence and did not increase the adverse event occurrence, indicating that Infliximab treatment is a promising therapeutic strategy for the management of CD patients.

\section{Methods}

This meta-analysis was performed in accordance with the guidelines of the Preferred Reporting Items for Systematic Reviews and Meta-Analyses (PRISMA) statement (http://www.prisma-statement.org/).

2.1. Search Strategy and Inclusion Criteria. On Jun $1^{\text {st }}, 2018$, we conducted a systematic search of PubMed, Medline, Embase, and Web of Science databases for randomized controlled trials or cohort studies published between Jun $1^{\text {st }}, 2000$ and Jun $1^{\text {st }}, 2018$ in English using the search terms "Infliximab" and "Crohn's disease". All the studies enrolled using this strategy were checked independently by two authors; the articles that met all inclusion criteria were enrolled in the meta-analysis. The inclusion criteria were as follows: (1) patients diagnosed with $\mathrm{CD}$ based on tissue biopsy and treated with Infliximab; (2) double-blind, randomized, placebo-controlled or prospective studies; and (3) the outcome measures regarding the rates of CD recurrence.

\subsection{Testing for Heterogeneity and Risk of Bias, and Sensitivity} Analysis. A Q and $\mathrm{I}^{2}$ test were performed to analyze the heterogeneity of the studies included in this meta-analysis. A Galbraith radial plot was produced to indicate the cause of heterogeneity in studies. Each study was represented as a single dot, with a central regression line through the plot. The Cochrane collaboration tool method was used to evaluate the quality of the included studies and the risk of bias, and the Egger test was performed, a linear regression method to evaluate the bias in publications in the meta-analysis. Sensitivity analysis was calculated to evaluate the stability and reliability of the results.

2.3. Data Extraction and Outcome Measures. In our systematic review of the literature, the following variables were extracted. Name of the study, drug regimen, type of ileocolonic resection, timing of initiation of treatment, recurrence definition and follow-up were included. The aim of the meta-analysis was to investigate the efficacy and safety of Infliximab for the prevention of recurrence in patients with CD across studies. The outcomes included in the metaanalysis were the rates of endoscopic recurrence and clinical recurrence.

2.4. Statistical Analysis. From the included studies in the analysis, we extracted the rates of endoscopic recurrence and clinical recurrence for Infliximab and the placebo, along with the corresponding 95\% CI. The overall summary effect sizes were estimated using a random-effects model if heterogeneity was $>50 \%$, and fixed-effects model was used if heterogeneity was $<50 \%$. Numeration data was presented as relative risk (RR) and a p value of $<0.05$ was considered to be statistically significant. Forest plots and data analysis were performed using Review Manager (RevMan version 5.3; Nordic Cochrane Centre, Copenhagen, Denmark) and STATA software (version 12.0; StataCorp, College Station, TX, USA).

\section{Results}

3.1. Study Selection. A search of the PubMed, MEDLINE, Embase, and Web of Science databases was conducted, and 7 prospective trials were enrolled, after excluding duplicated, irrelevant, and nonfull text articles. The systematic search identified 413 original articles; the flow diagram for the identified studies is shown in Figure 1. A total of 49 duplicates, 2 abstracts, and 354 irrelevant articles were excluded as they did not meet the inclusion criteria for this meta-analysis. Finally, 7 prospective trials were included in the metaanalysis. All studies reported quantitative analysis regarding the Infliximab in patients with CD. All were published in English.

3.2. Study Characterization. This systematic review included the data of 455 patients diagnosed with CD. A total of 7 prospective trials were enrolled in the systematic review. All studies had control groups in which the baseline of CD severity did not significantly differ from the treatment group. The characteristics of the cohort studies are shown in Table 1 . In the 7 prospective trials, all the studies considered Infliximab monotherapy, and all the patients in the treatment group received Infliximab $5 \mathrm{mg} / \mathrm{kg}$ once a day. The timing of initiation of treatment ranged from 2 to 8 weeks. Sorrentino et al. (2007) and Fukushima et al. (2018) were designed for 24 months, and other trials were designed for 12 months. In Sorrentino et al. (2007), Regueiro et al. (2009), Yoshida et al. (2012), and Regueiro et al. (2016) trials, the type of ileocolonic resection was macroscopically diseased bowel resected, and in Armuzzi et al. (2013), Tursi et al. (2014), and Fukushima et al. (2018) studies, curative ileocolonic resection was made. Moreover, the clinical recurrence definition in the 7 studies was based on Hanauer, CDAI or HBI criteria, while the endoscopic recurrence of all the trials was defined as Rutgeerts score $\geq 2$. The characteristics of the included trials are shown in Table 1. 


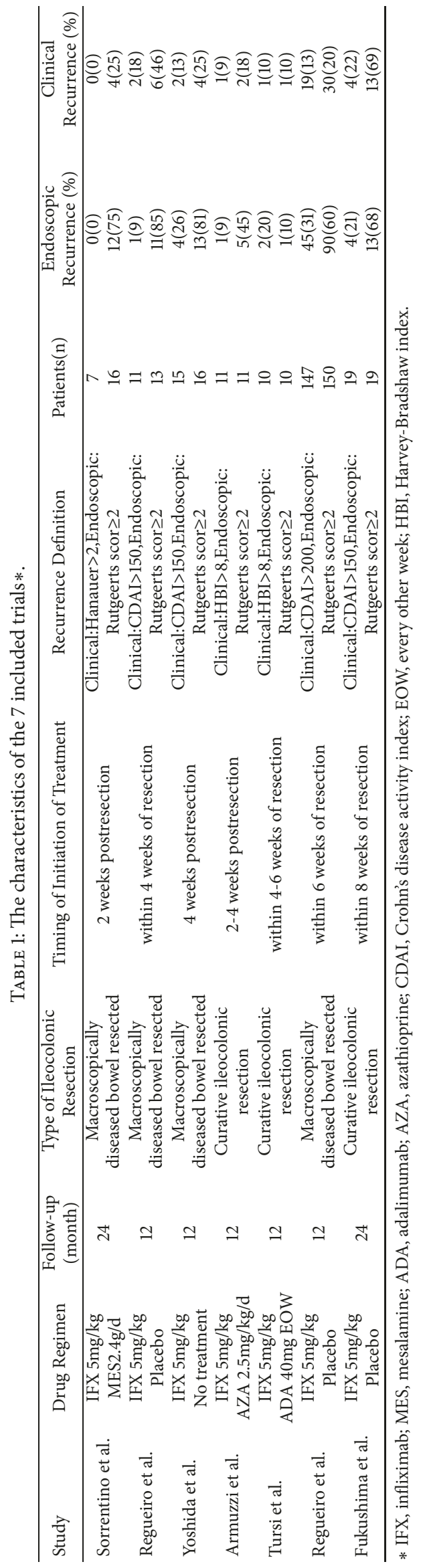




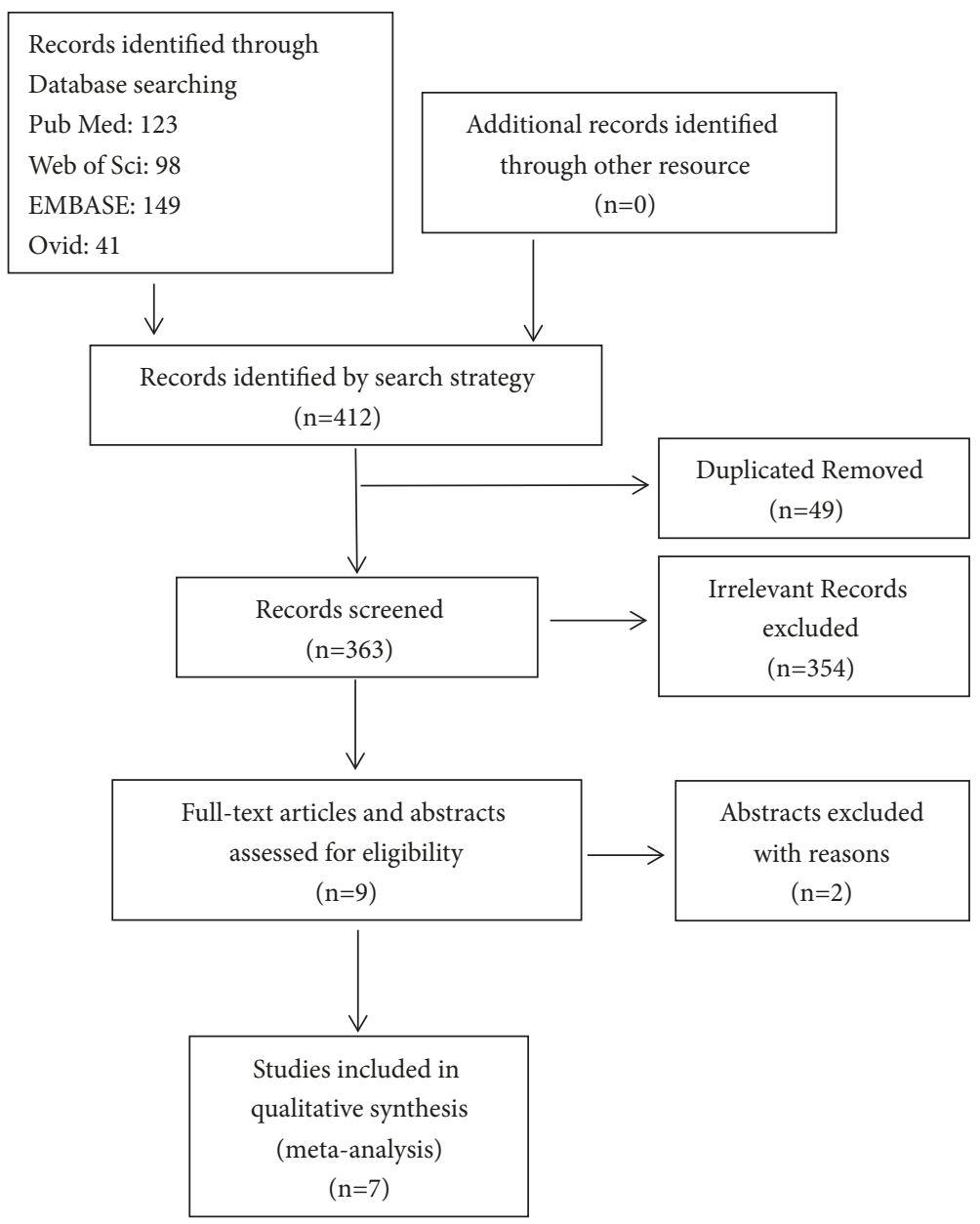

FIGURE 1: Flow diagram of study selection. A total of 455 potentially relevant studies were collected, of which 7 prospective trials were included in the meta-analysis.

3.3. Risk of Bias, Sensitivity Analysis, and Quality Assessment. A Funnel plot was produced to describe the bias in publication of the 7 prospective trials (Figure 2). All studies enrolled in the meta-analysis exhibited a low risk of bias. The Cochrane collaboration tool was used to identify the risk of bias of 7 prospective trials, as shown in Figure 3. All studies applied random sequence generation, allocation concealment, blinding of participants and personnel, and blinding of outcome assessment and had a low risk of incomplete outcome data and selective reporting. The clinical outcome data in the included studies were based on existing guidelines, and their baselines were similar. A Funnel plot and Egger test showed there was no significant bias in the cohort studies $(p>0.05)$. A sensitivity analysis plot was performed to analyze the sensitivity of the trials included in this meta-analysis, which indicated that no studies needed to be excluded (Figure 4).

3.4. Efficacy Outcomes of Infliximab. In a pooled analysis of the 7 prospective trials, a significant improvement in efficacy for Infliximab versus the placebo was observed in various clinical indexes: rates of endoscopic recurrence and clinical recurrence.

3.4.1. Rates of Endoscopic Recurrence. Endoscopy is gold standard for monitoring postoperative recurrence and endoscopic recurrence is defined as Rutgeerts score $\geq 2$. Higher rates of endoscopic recurrence indicate worse curative effect. In the pooled analysis, Infliximab therapy significantly decreased the rate of endoscopic recurrence of CD compared with a placebo $(\mathrm{RR}=0.421 ; 95 \%$ CI 0.328 to $0.539 ; p<0.001$; Figure 5(a)). Except for the Tursi et al. (2014) study, all the trials showed a substantial reduction in rates of endoscopic recurrence.

3.4.2. Rates of Clinical Recurrence. As endoscopy is an invasive examination so that it is difficult to be used as a routine means of close follow-up after surgery, clinical examination strategy helps to improve compliance of patients after operation and clinical recurrence is defined as Hanauer $>2$, $\mathrm{CDAI}>150$, or $\mathrm{HBI}>8$. A random-effect model was used to perform the meta-analysis of the six included prospective 


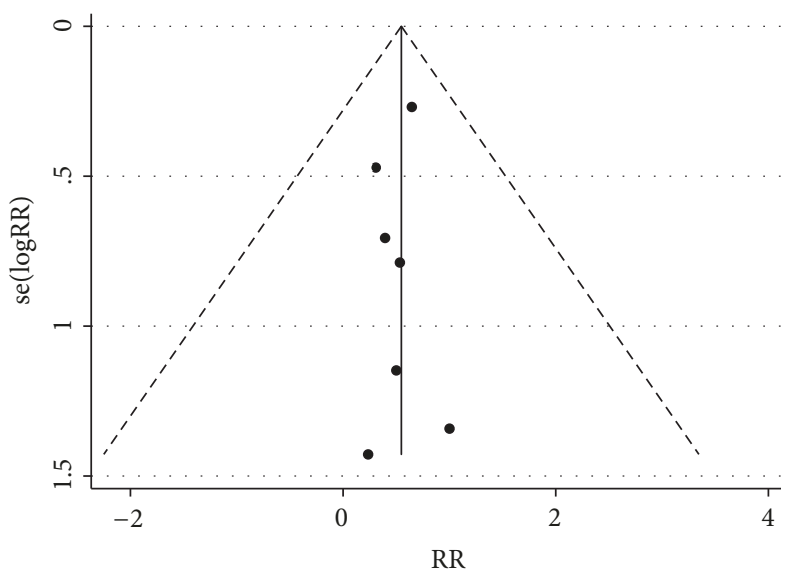

Figure 2: Risk of publication bias for each trial. Both the Funnel plot and Egger test showed no significant evidence of bias in the publication.

trials. There was a significant reduction of the rates of clinical recurrence in the Infliximab-treated group compared with the placebo group $(\mathrm{RR}=0.519 ; 95 \%$ CI 0.349 to $0.774 ; p=0.001$; Figure 5(b)).

3.5. Safety of Infliximab Treatment. Overall, Infliximab was well tolerated and has a favorable safety profile that is substantially better than most systemic agents. The most commonly reported adverse events (AE) in patients treated with Infliximab were lupus-like reaction, infusion reaction, bronchitis, nasopharyngitis, pyelonephritis, severe abdominal pain, and abdominal wall abscess. We found that AE occurred at a similar rate among different trials without statistical significance $(\mathrm{RR}=0.551 ; 95 \% \mathrm{CI} 0.379-0.800 ; p=0.002$, Figure 6(a)). Finally, similar risk of study discontinuation between Infliximab and placebo groups was observed $(\mathrm{RR}=0.983 ; 95 \%$ CI 0.660 1.466; $p=0.934$, Figure 6(b)).

3.6. Test of Heterogeneity. We attempted to identify potential sources of heterogeneity in the overall effect based on using forest plots. We discovered that the rates of endoscopic recurrence $\left(\mathrm{I}^{2}=28.7 \%, \mathrm{p}=0.210\right)$ slightly affected the heterogeneity of the outcome result, whereas the rates of clinical recurrence $\left(\mathrm{I}^{2}=0.0 \%, \mathrm{p}=0.858\right)$, the adverse events occurrence $\left(\mathrm{I}^{2}=0.0 \%\right.$, $\mathrm{p}=0.590)$, and the rates of study discontinuation $\left(\mathrm{I}^{2}=0.0 \%\right.$, $\mathrm{p}=0.976$, Figure 7 ) were not statistically heterogeneous. To explore the source of heterogeneity, a Galbraith radial plot was performed. The results showed that no study was the major source of heterogeneity.

\section{Discussion}

The results of this meta-analysis demonstrated the efficacy and safety of Infliximab in the prevention of recurrence in patients with $\mathrm{CD}$ across 7 prospective trials. Infliximab was shown to be consistently more effective than a placebo. To our knowledge, this is the most recent systematic analysis and comparison of all Infliximab study data for the prevention of $\mathrm{CD}$ recurrence.

The trials included had a high level of consistency in the patients enrolled, randomization and masking, and efficacy outcomes. The endpoints these clinical trials applied included both objective and subjective indexes, providing comprehensive assessment of the treatment responses. Endoscopic recurrence is an objective assessment of $\mathrm{CD}$ recurrence, whereas clinical recurrence combines both objective and subjective evaluations of the disease recurrence. In most studies clinical recurrence after the resective surgery is characterized by CDAI $>220$ and in the Regueiro et al. 2009, Yoshida et al. 2012, and Fukushima et al. 2018 studies the criteria are defined as CDAI $>150$. The 7 trials included in this review were consistent in applying similar objective and subjective recurrence rates, and the results from 7 trials were also consistent, in that Infliximab was effective in the prevention of CD recurrence as evidenced not only by decreased rates of endoscopic recurrence, but also by decreased rates of clinical recurrence.

In our meta-analysis, the sample size was not large enough for valuable statistical results; however, there was a trend towards the efficacy of Infliximab treatment. Further trials should be performed to optimize the dose for the best cost/effect of Infliximab. In addition, a combination of Infliximab with other drugs may improve its effect and thus decrease the dose of Infliximab while achieving similar efficacy. Prospective trials with Infliximab did not identify any serious adverse events. Further clinical trials could provide more information on this issue. Overall, Infliximab is a promising therapeutic agent for improving the management of CD patients. Most importantly, the Infliximab treatment was demonstrated to be very safe.

A limitation of this meta-analysis is the limited number of trials included. However, all the trials were prospective studies and are therefore of high quality. Furthermore, although almost all the publications included in this study were from 


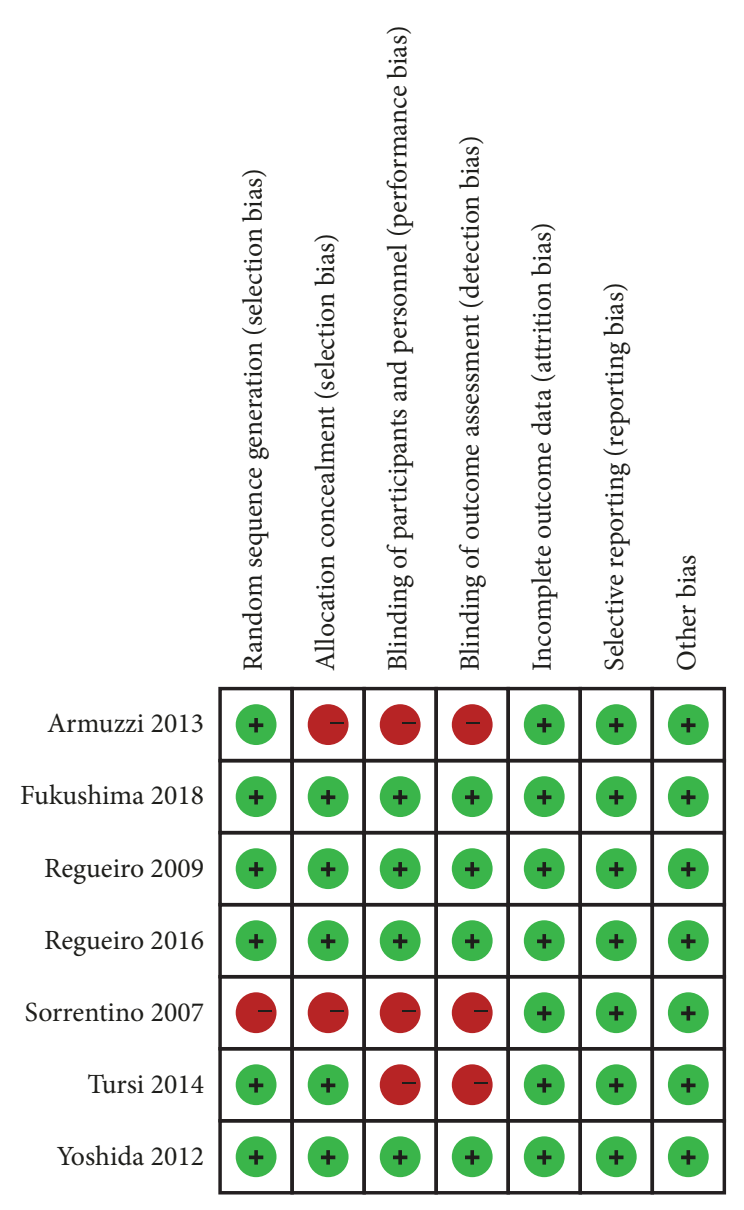

FIGURE 3: Risk of bias assessment. The qualities of the included studies were evaluated using Cochrane collaboration tool, indicating that there is no significant risk of bias in the included trials of this meta-analysis.

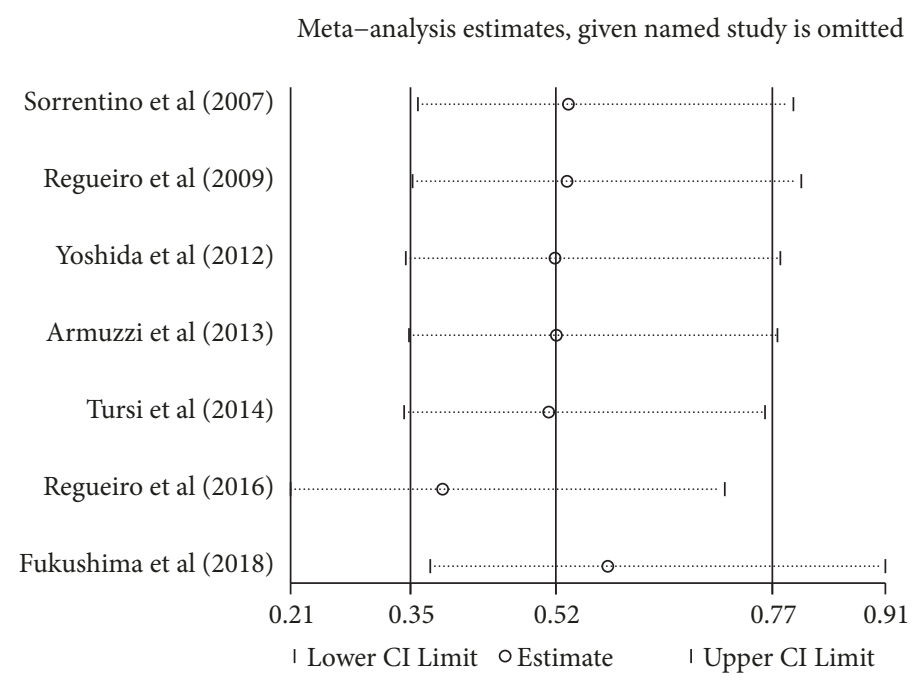

FIGURE 4: Sensitivity analysis plot. Hollow circles stand for relative risk (RR) of included trials, and Horizontal lines represent 95\%Cls of the RR. Almost all the trials were within the two regression lines. 


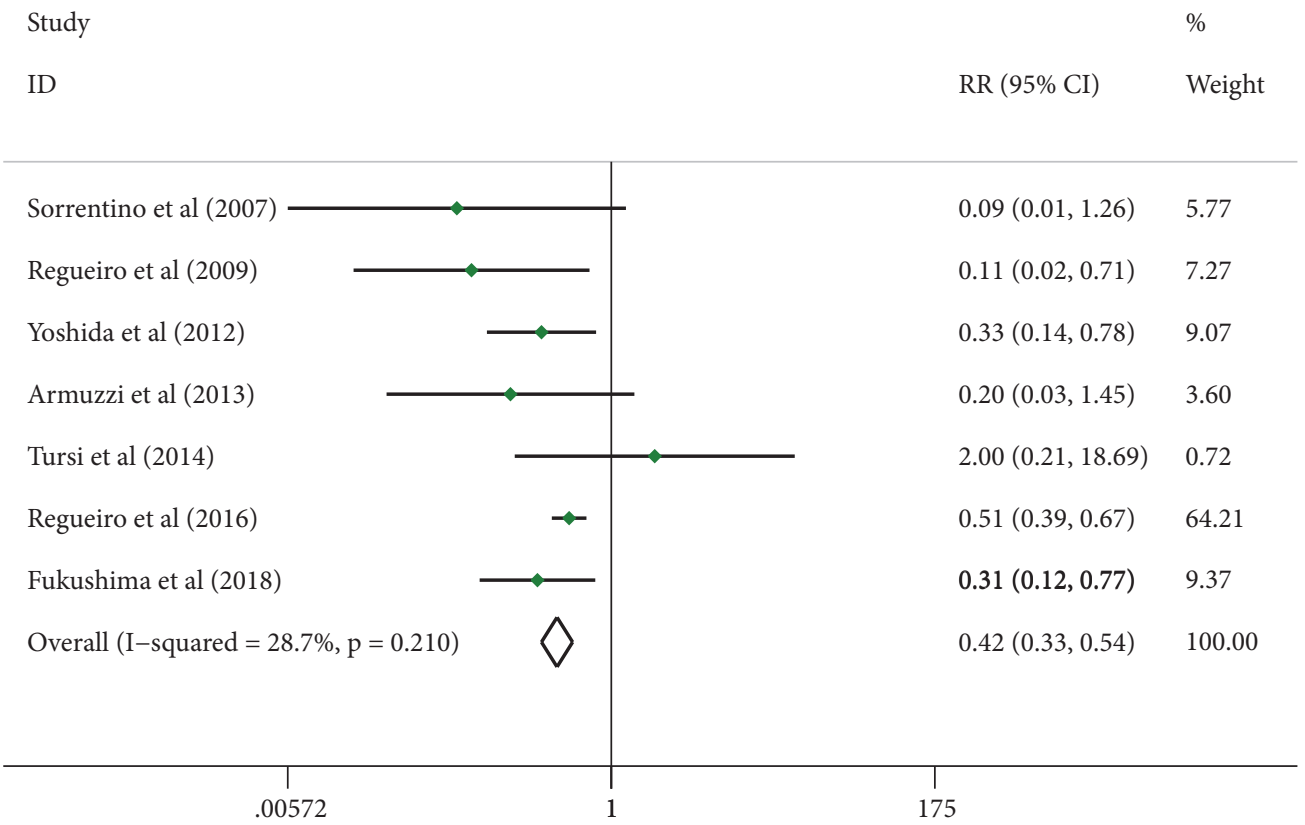

(a)

Study

ID

\begin{tabular}{|c|c|c|}
\hline Sorrentino et al (2007) - & $0.24(0.01,3.88)$ & 4.97 \\
\hline Regueiro et al (2009) & $0.39(0.10,1.57)$ & 9.49 \\
\hline Yoshida et al (2012) & $0.53(0.11,2.50)$ & 6.68 \\
\hline Armuzzi et al (2013) & $0.50(0.05,4.75)$ & 3.45 \\
\hline Tursi et al (2014) & $1.00(0.07,13.87)$ & 1.73 \\
\hline Regueiro et al (2016) & $0.65(0.38,1.10)$ & 51.25 \\
\hline Fukushima et al (2018) & $0.31(0.12,0.77)$ & 22.43 \\
\hline Overall $(\mathrm{I}-\mathrm{squared}=0.0 \%, \mathrm{p}=0.858)$ & $0.52(0.35,0.77)$ & 100.00 \\
\hline .0144 & 9.5 & \\
\hline
\end{tabular}

(b)

$\%$
RR $(95 \%$ CI $) \quad$ Weight

.

FIGURE 5: Forest plot of the efficacy of Infliximab in the 7 prospective trials. (a) Rates of endoscopic recurrence. (b) Rates of clinical recurrence. Green dots represent the RR of rates of endoscopic recurrence and blue dots represent the RR of rates of clinical recurrence. Horizontal lines represent $95 \% \mathrm{Cls}$ of the RR estimates. Diamonds stand for summary effect estimate of the meta-analysis.

top journals with high impact factors, risks of bias, e.g., due to funding from the pharmaceutical industry, may exist. Due to ethical limitations, it is difficult to perform clinical trials on healthy people, which play important role in control group, and it would be fine to compare low-risk population (the first resection/nonpenetrating disease) and high-risk CD populations on the efficacy of Infliximab (very relevant for clinical practice); future studies should address the issue in these patient's groups.

Systemic immunosuppressant drugs are still recommended by the current guidelines for the prevention of $\mathrm{CD}$ recurrence when topical treatment or phototherapy is 
Study

ID

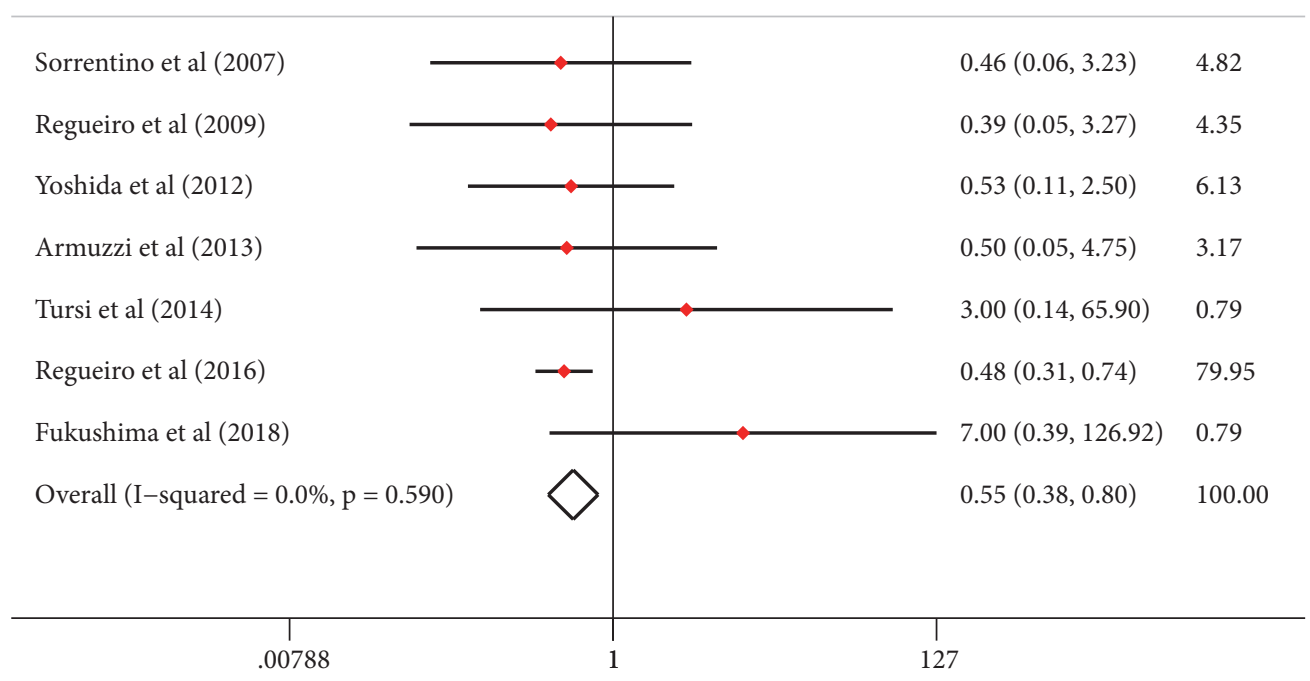

(a)

$$
\text { Study }
$$

ID
$\%$

RR (95\% CI) Weight

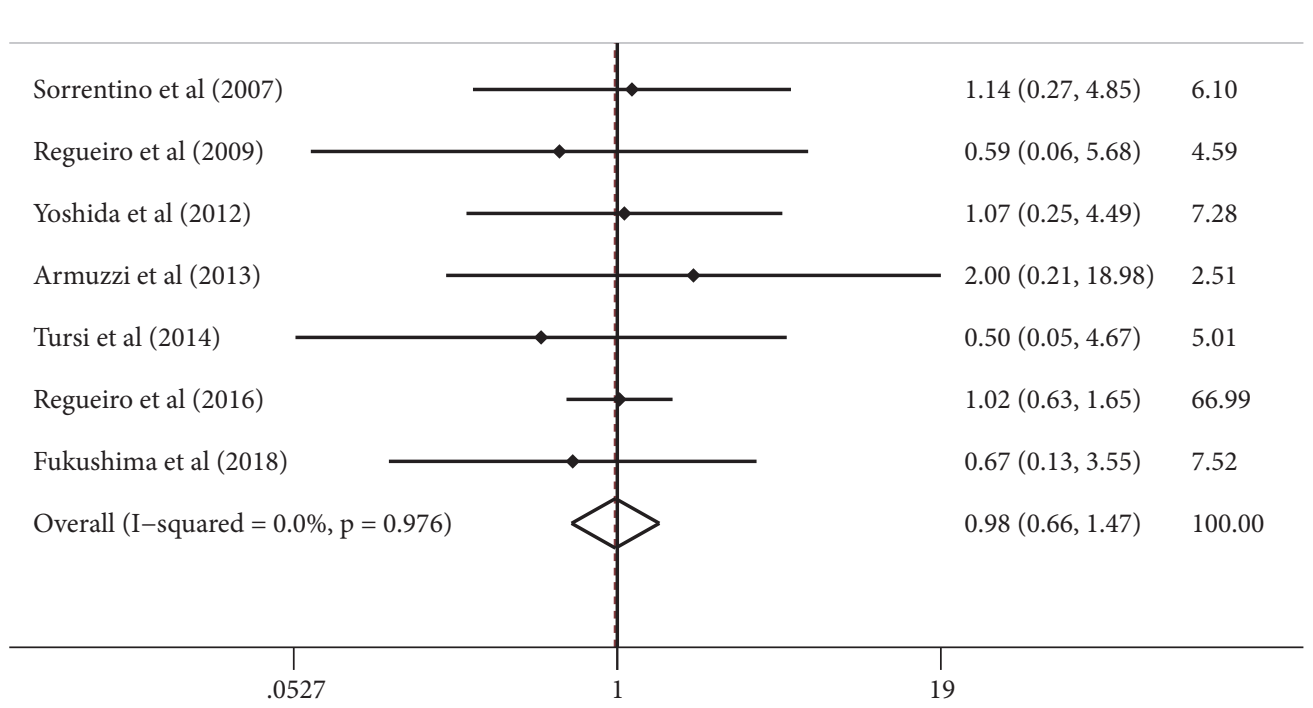

(b)

FIGURE 6: Forest plot of the safety of Infliximab treatment. (a) Rates of adverse events. (b) Rates of study discontinuation. Red dots represent the RR of rates of adverse events and black dots represent the RR of rates of study discontinuation. Horizontal lines represent $95 \%$ Cls of the RR estimates. Diamonds stand for summary effect estimate of the meta-analysis.

ineffective; however, the frequency of serious adverse events hinders the use of systemic anti-inflammation treatment. Our findings clearly indicate that Infliximab is promising as an anti-CD recurrence medication and meets the requirements of treating the disease specifically and efficiently. Further investigations should aim to prove the long-term stability, efficacy, and safety of Infliximab in the treatment of CD.

\section{Conflicts of Interest}

The authors state no conflicts of interest. 


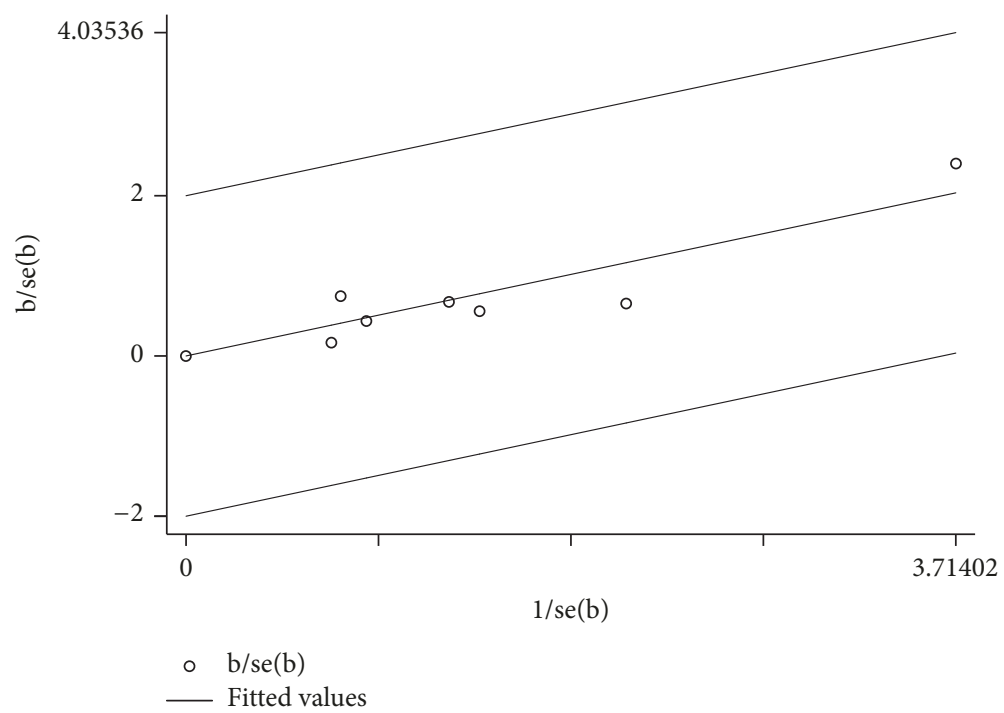

FIgURE 7: Galbraith radial plot. The figure shows the contribution of results from the 7 studies to the heterogeneity. No study was shown to be the source of the heterogeneity.

\section{Acknowledgments}

The article was funded with National Natural Science Foundation of China (No. 81603622) and Natural Science Foundation of Jiangsu Province of China (No. BK20161319).

\section{References}

[1] D. C. Baumgart and W. J. Sandborn, "Crohn's disease," The Lancet, vol. 380, pp. 1590-1605, 2012.

[2] A. P. Yu, L. A. Cabanilla, E. Q. Wu et al., "The costs of Crohn's disease in the United States and other Western countries: a systematic review," Current Medical Research and Opinion, vol. 24, pp. 319-328, 2008.

[3] J. Cosnes, I. Nion-Larmurier, L. Beaugerie, P. Afchain, E. Tiret, and J.-P. Gendre, "Impact of the increasing use of immunosuppressants in Crohn's disease on the need for intestinal surgery," Gut, vol. 54, no. 2, pp. 237-241, 2005.

[4] A. Buisson, J. Chevaux, P. B. Allen, G. Bommelaer, and L. Peyrin-Biroulet, "Review article: the natural history of postoperative Crohn's disease recurrence," Alimentary Pharmacology \& Therapeutics, vol. 35, no. 6, pp. 625-633, 2012.

[5] M.-S. Ong, R. J. Grand, and K. D. Mandl, "Trends in Pharmacologic Interventions for Preventing Recurrence of Crohn's Disease after Ileocolonic Surgery," Inflammatory Bowel Diseases, vol. 22, no. 10, pp. 2432-2441, 2016.

[6] F. Takeshima, D. Yoshikawa, S. Higashi et al., "Clinical efficacy of adalimumab in Crohn's disease: A real practice observational study in Japan," BMC Gastroenterology, vol. 16, no. 1, article no. 82, 2016.

[7] W. Reinisch, S. Angelberger, W. Petritsch et al., "Azathioprine versus mesalazine for prevention of postoperative clinical recurrence in patients with Crohn's disease with endoscopic recurrence: Efficacy and safety results of a randomised, doubleblind, double-dummy, multicentre trial," Gut, vol. 59, no. 6, pp. 752-759, 2010.

[8] M. Collins, H. Sarter, C. Gower-Rousseau et al., "Previous Exposure to Multiple Anti-TNF Is Associated with Decreased
Efficiency in Preventing Postoperative Crohn's Disease Recurrence," Journal of Crohn's and Colitis, vol. 11, no. 3, pp. 281-288, 2017.

[9] E. Savarino, G. Bodini, P. Dulbecco et al., "Adalimumab is more effective than azathioprine and mesalamine at preventing postoperative recurrence of Crohn's disease: A randomized controlled trial," American Journal of Gastroenterology, vol. 108, no. 11, pp. 1731-1742, 2013.

[10] P. De Cruz, M. A. Kamm, A. L. Hamilton et al., "Efficacy of thiopurines and adalimumab in preventing Crohn's disease recurrence in high-risk patients-A POCER study analysis," Alimentary Pharmacology \& Therapeutics, vol. 42, pp. 867-879, 2015.

[11] D. Sorrentino, G. Terrosu, C. Avellini, and S. Maiero, "Infliximab with low-dose methotrexate for prevention of postsurgical recurrence of ileocolonic Crohn disease [1]," JAMA Internal Medicine, vol. 167, no. 16, pp. 1804-1807, 2007.

[12] M. Regueiro, W. Schraut, L. Baidoo et al., "Infliximab Prevents Crohn's Disease Recurrence After Ileal Resection," Gastroenterology, vol. 136, no. 2, pp. 441-el, 2009.

[13] K. Yoshida, K. Fukunaga, H. Ikeuchi et al., "Scheduled infliximab monotherapy to prevent recurrence of Crohn's disease following ileocolic or ileal resection: A 3-year prospective randomized open trial," Inflammatory Bowel Diseases, vol. 18, no. 9, pp. 1617-1623, 2012.

[14] A. Armuzzi, C. Felice, A. Papa et al., "Prevention of postoperative recurrence with azathioprine or infliximab in patients with Crohn's disease: An open-label pilot study," Journal of Crohn's and Colitis, vol. 7, no. 12, pp. e623-e629, 2013.

[15] A. Tursi, W. Elisei, M. Picchio et al., "Comparison of the effectiveness of infliximab and adalimumab in preventing postoperative recurrence in patients with Crohn's disease: an openlabel, pilot study," Techniques in Coloproctology, vol. 18, no. 11, pp. 1041-1046, 2014.

[16] M. Regueiro, B. G. Feagan, B. Zou et al., "Infliximab reduces endoscopic, butnotclinical, recurrence of Crohn's disease after ileocolonic resection," Gastroenterology, vol. 150, pp. 1568-1578, 2016. 
[17] K. Fukushima, A. Sugita, K. Futami et al., "Postoperative therapy with infliximab for Crohn's disease: a 2-year prospective randomized multicenter study in Japan," Surgery Today, vol. 48, no. 6, pp. 584-590, 2018.

[18] E. Scapa, N. Maharshak, Y. Kariv et al., "Sal150 Early Initiation of Adalimumab Significantly Diminishes Post-Operative Crohn's Disease Recurrence, and Is Superior to Immunomodulator Therapy. Preliminary Results From the POPART Trial," Gastroenterology, vol. 148, no. 4, pp. S-240-S-241, 2015.

[19] Y. Qiu, R. Mao, B. Chen, Y. He, Z. Zeng, and M. Chen, "Systematic Review with Meta-analysis of Prospective Studies: Anti-tumour Necrosis Factor for Prevention of Postoperative Crohn's Disease Recurrence," Journal of Crohn's and Colitis, vol. 9, no. 10, pp. 918-927, 2015.

[20] A. Carla-Moreau, S. Paul, X. Roblin, C. Genin, and L. PeyrinBiroulet, "Prevention and treatment of postoperative Crohn's disease recurrence with anti-TNF therapy: A meta-analysis of controlled trials," Digestive and Liver Disease, vol. 47, no. 3, pp. 191-196, 2015.

[21] S. Singh, S. K. Garg, D. S. Pardi, Z. Wang, M. H. Murad, and E. V. Loftus, "Comparative efficacy of pharmacologic interventions in preventing relapse of Crohn's disease after surgery: A systematic review and network meta-analysis," Gastroenterology, vol. 148, no. 1, pp. 64-76, 2015. 

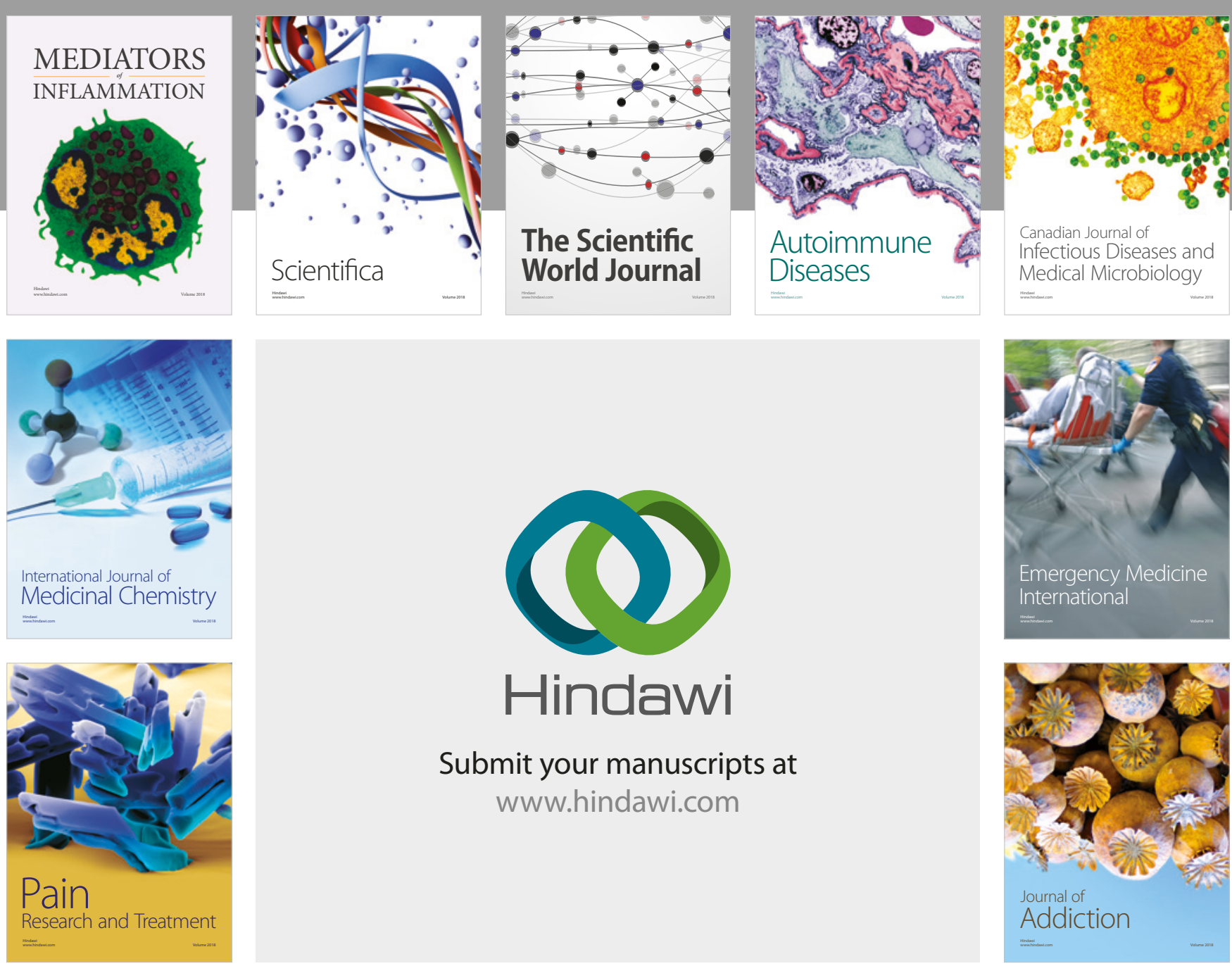

Canadian Journal of
Infectious Diseases and Medical Microbiology

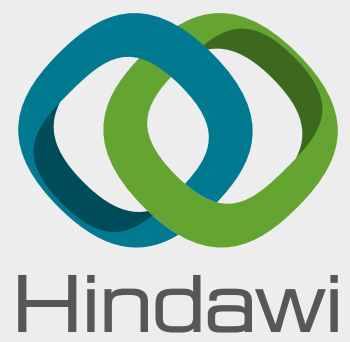

Submit your manuscripts at

www.hindawi.com
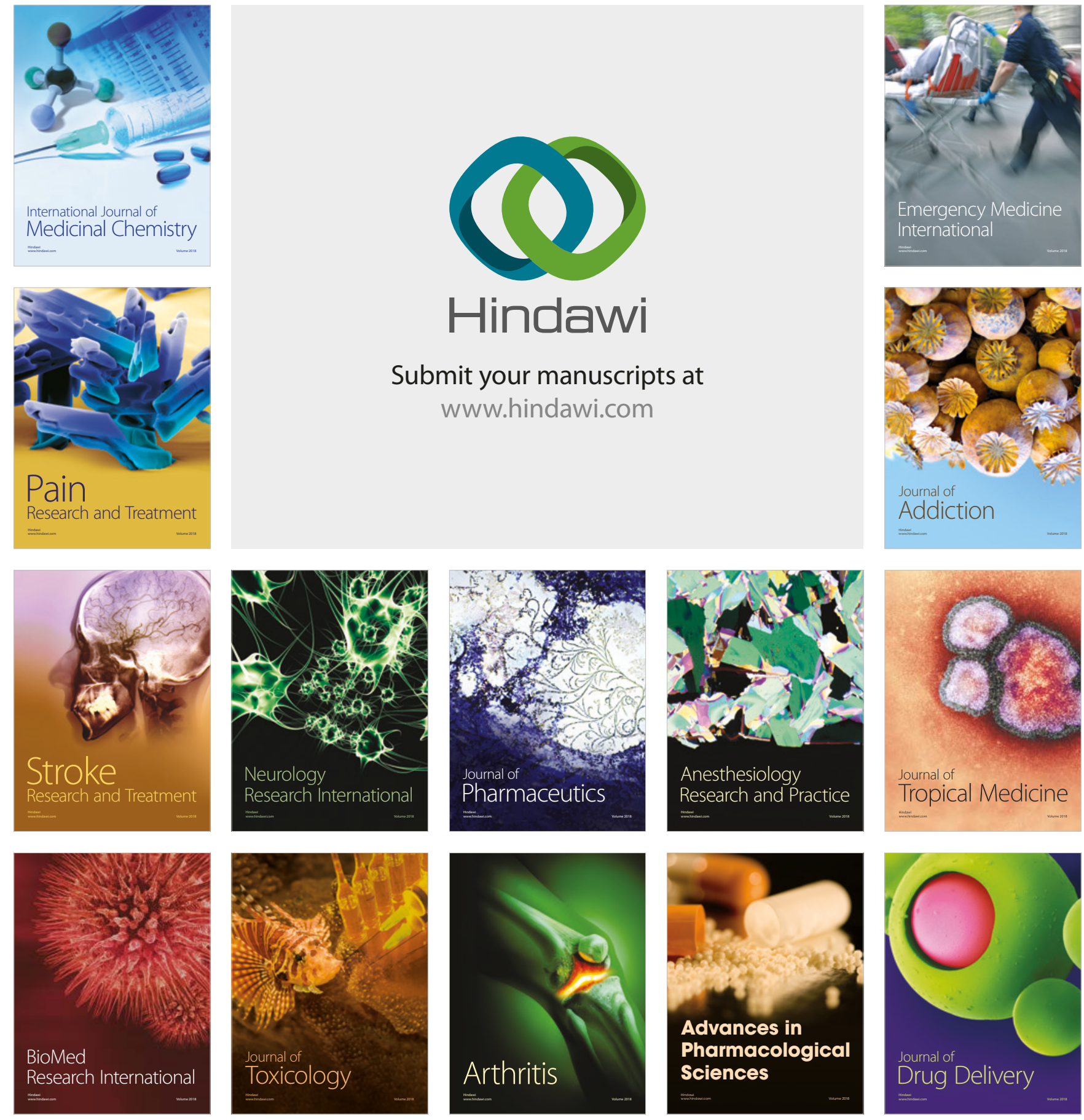